\title{
Control of Permanent Magnet Synchronous Motors using Observer Based MIMO Feedback Linearization
}

\author{
Gh. Omrani, B. Abdi, V. Behnamgol* and Gh. Derakhshan \\ Islamic Azad University, Damavand Branch, Department of Electrical Engineering, Faculty of Control Engineering, Damavand, Iran.
}

Received 7 March 2021; Accepted 19 June 2021

\begin{abstract}
Permanent magnet synchronous motors are one of the most widely used motors in various industries. By using a closedloop control system, the speed control of these motors can be adjusted. The dynamics of this type of motor are nonlinear. In recent years, nonlinear controllers have been used to control permanent magnet synchronous motors. Although these methods do not require linearization around the operating point, they are not robust to uncertainty. The use of an observerbased nonlinear controller is a solution for controlling uncertain nonlinear systems. In this method, the uncertainty estimation is provided to the controller using an extended observer. Another point in controlling a permanent magnet synchronous motor is the two-input two-output of the dynamic model of this system. Therefore, the use of multivariate control techniques to design the control input vector would be desirable. In this paper, an observer-based nonlinear vector method is used to control the MIMO model of the permanent magnet synchronous motor. For this purpose, we assume the load torque to be uncertain and estimate it using an extended observer. Then, the MIMO feedback linearization method is used to design the control input vector. Using the proposed method has advantages such as a nonlinear control system, robust to uncertainty, and vector controller scheme. These advantages are demonstrated by analytically and using computer simulations.
\end{abstract}

Keywords: Permanent Magnet Synchronous Motor, MIMO Feedback Linearization, disturbance observer.

\section{Introduction}

Industrial motors can be divided into two categories: direct current (DC) and alternating current (AC). Also, alternating current motors can be divided into two categories: synchronous and asynchronous. Permanent magnet synchronous motors (PMSM) are classified as alternating current and synchronous motors. Today, PMSMs have a very appropriate position in the industry due to their high efficiency, high power density and wide speed range, and they are used in industries such as automobile manufacturing, home appliances, aerospace and marine industries. These motors are a very suitable option for control applications due to their low inertia and good controllability. The simple structure of these motors frugality their repair and maintenance cost and time [38]. The first step in controlling PMSMs is to achieve a dynamic model. In recent years, modeling of this category of engines has been done in authentic references and can be cited. In many references, a common model in d-q coordinates has been used for these motors. For example, in reference [12], modeling of PMSMs is performed and dynamic equations are given in $\mathrm{d}-\mathrm{q}$ coordinates. Also in the reference [1] by considering the three variables of id, iq currents and shaft angular speed, the nonlinear dynamics of this system is obtained. In references [29] and [2] similar to the reference [1] by defining the state variables in $\mathrm{d}$-q coordinates, the nonlinear state equations of the PMSM are presented. Uncertainty and disturbance due to load torque changes are also modeled in these references. In the reference [22] a similar model has been obtained with the

*E-mail address: vahidbehnamgol@gmail.com ISSN: 1791-2377 @ 2021 School of Science, IHU. All rights reserved.

doi:10.25103/jestr.143.04 mentioned references, but in this reference, the size of the inductors in the $\mathrm{d}$ and $\mathrm{q}$ directions has been equalized and simplification has been done. In most of these references, the voltage in the $\mathrm{d}$ and $\mathrm{q}$ directions is assumed to be two control inputs. In the reference [30] in the d-q coordinates, a different model is presented by considering the flux instead of the current as a state variable. The reference [30] is similar to the previous references and in each equation, the state of changes of electrical and mechanical parameters and load torque is assumed to be uncertain. Finally, the linearization of equations has been used to control it. In the reference [22] a fractional-order model for nonlinear dynamics of a PMSM is considered. In [30] modeling has been done with the aim of integrated position and speed control for a PMSM, and the variables of current state, position and speed of the motor shaft have been considered.

Methods of control of PMSMs are divided into two groups: vector and scalar method. Using vector control method, the high performance of three-phase PMSM can be achieved. In this method, the current vectors $i_{d}$ and $i_{q}$ are controlled. The field control achieved by zeroing the $i_{d}$ current causes the magnet to adjust the magnet on the $d$ axis and creates a linear relationship between current and torque [10]. In controlling the vector magnetism of PMSMs, it has been tried that the rotor flux is always on the $\mathrm{d}$ axis and the stator current flux generation component $i_{d s}$ should be zero so that the torque does not decrease. With the stator current being zero in the vector control method, the torque is adjusted by the other component of the current. A speed sensor connected to the rotor shaft must be used to control the speed [23]. The most common method of controlling industrial systems is the proportional controller of the integral derivative. This 
controller has been used to control the speed of a PMSM [16]. The dynamics of these motors are nonlinear and PID linear controllers cannot have good control in all operating conditions of the motor, so it cannot be a good option for accurate control of the speed of PMSMs. Using a PID controller with constant coefficients has been a good option for applying control commands. Based on this, the reference [28] provides a self-adjusting PID controller for the PMSM. In reference [5] using the optimal particle aggregation smart method, the PID controller parameters are adjusted to control the PMSM. In [25] using a fuzzy controller, the parameters of the PID controller are set based on the change of engine speed and online. Reference [8] using neural networks, an algorithm is proposed to control and adjust the parameters of the PID controller, which can detect parametric changes and increase the robustness of the system. Create and measure a good controller for synchronous motor speeds of three-phase permanent magnet in the presence of noise and environmental disturbances. In references [13] and [24] using the adaptive control method, the control of the PMSM has been done. In reference [14] the model predictive control has been used to control the speed of the three-phase PMSM. In [29], adaptive sliding mode control is used to minimize torque ripple in the PMSM. In this method, chattering is the main problem of sliding mode in the control signal prevents implementation. In [22], a fuzzy first order sliding mode hybrid controller is used to control the system, and in [30] integrated position and speed control for a PMSM using sliding mode control optimized with smart algorithms done. One solution to reduce undesirable fluctuations in sliding mode control is to use a disturbance observer. The design principles of disturbance observer are presented in [11], [21], [6] and [17]. In [1], a terminal-order non-singular sliding mode controller based on the observer is used to control the PMSM. In this reference, the observer is used to estimate the position and speed of the motor shaft, and control input equation includes the sign function. Therefore, the saturation function has been used to prevent chattering from occurring, but it still reduces accuracy. In [2] a second-order sliding mode controller is used for the nonlinear and uncertain system of the PMSM in d-q coordinates, which emits a smooth control signal. This controller requires an uncertain exact estimate.

In [22] sliding mode controller based on an extended observer is used to control the speed of the PMSM. In this reference, the slider variable is defined based on the terminal sliding mode method, which guarantees the time-limited convergence of state variables. The problem with this method is the use of the discontinuous function of the signal in the control input relation, which is solved using the continuous approximation method and the saturation function replaces the signal function. In [29] the sliding mode-predictor hybrid controller is used along with an extended observer to estimate the disturbance, and there is also the problem of chattering due to the signal function. Reference [27] uses the ultimate sliding mode control to design the controller and the nonlinear viewer to estimate the uncertainties. The only problem with this reference, according to the principles of first-order sliding mode, is the use of the discontinuous function of the signal and the creation of chattering in the control signal. Nonlinear systems can be linearized without linearization and approximation using the linearizer feedback method. Then, for the linearized system, the controller can be designed from any linear method. In reference [9], the design principles are presented by the linear feedback method. The design of nonlinear controllers for multi-input multi-output systems is also examined in references [31] and [32]. In the linear feedback method for single-input single-output systems and multi-input multi-output systems, first, the system is converted to a linear form by removing nonlinear sentences, and then for the linearized system, the controller can be used with any designed a linear method. A combination of control theories and multivariate techniques has also been proposed by researchers for PMSMs. In [33, 35] fuzzy-multivariate hybrid controller in [34] optimal-multivariate hybrid control and in [36] predictive-multivariate linear control is designed for PMSM. By studying the results of references, in many references, controllers of the non-linear type such as sliding mode and nonlinear $H_{\infty}$ are designed for this system. In recent years, extended observer nonlinear controllers have been introduced for uncertain nonlinear systems and have many advantages over robust nonlinear control methods such as slip mode and nonlinear $H_{\infty}$. These motors have two inputtwo output equations, so to increase the efficiency of the control system, it is better to use multivariate control theories for it. Summarizing the contents mentioned as an innovation of this paper, the design of a nonlinear controller based on the observer in vector state for a two-input two-output system of a PMSM is proposed, which will be a hybrid method.

In this section, the research topic is described. Also, researches on the control of PMSMs were reviewed. In the second part, the concepts related to PMSMs and its modeling will be explained. In the third section, the proposed control theory is described and this method will be used in the fourth section to control the PMSM. In the fifth section, the simulation results are presented and finally in the last section, we summarize and conclude.

\section{Dynamic model of PMSM}

In reference [3], the equations of state space are in the form of equation (1).

$$
\left\{\begin{array}{l}
i_{d}=-\frac{R_{S}}{L} i_{d}+P_{n} \omega i_{q}+\frac{u_{d}}{L} \\
i_{q}=-P_{n} \omega i_{d}-\frac{R_{S}}{L} i_{q}-\frac{P_{n} \psi_{f}}{L} \omega+\frac{u_{q}}{L} \\
\dot{\omega}=\frac{P_{n} \psi_{f}}{J} i_{q}-\frac{B}{J} \omega-\frac{T_{L}}{J}
\end{array}\right.
$$

where $I_{d}$ and $I_{q}$ are the currents of the d-q axes, $\psi_{f}$ is the leakage flux between the rotor and the stator, $\mathrm{u}_{\mathrm{d}}$ and $\mathrm{u}_{\mathrm{q}}$ are the voltages applied to the motor at reference $d-q$ and $\omega$ the rotor speed, $L_{d}$ and $L_{q}$ are the inductances of the $d-q$ axes, $R_{s}$ is the stator resistance, $P_{n}$ is the number of pairs of motor poles and $\mathrm{T}_{\mathrm{L}}$ is the load torque that is assumed to be uncertain. In this reference, $\mathrm{L}_{\mathrm{d}}$ and $\mathrm{L}_{\mathrm{q}}$ are equal.

\section{Proposed control theory}

\subsection{MIMO feedback linearization}

Feedback linearization is one of the nonlinear control theories for controlling dynamic systems. First, the nonlinear system is transformed into a new dynamic system using a nonlinear feedback, then linear control methods can be used to control this linear system. This feedback can be designed in two ways: input-state and input-output. To stabilize a nonlinear system, the state-input linear feedback is used to stabilize a nonlinear system and input-output linear feedback theory is used for tracking. For the input-state linear feedback theory, consider the following nonlinear system: 
$\dot{x}=A x+B w(x)[u-\phi(x)]$

$x \in X \subset \mathbb{R}^{n}$ are state vectors, $u \in \mathbb{R}$ are control inputs, $A \in$ $\mathbb{R}^{n \times n}, B \in \mathbb{R}^{n \times 1}$ are coefficient matrices, and $\phi(x), w(x): D \subset \mathbb{R}^{n} \rightarrow \mathbb{R}$ are nonlinear functions. $w(x) \neq 0$ and $(A, B)$ are assumed to be controllable. In the input-state linear feedback method, the control input is designed as follows:

$$
u=\phi(x)+w^{-1}(x) v
$$

The closed loop system will be in the following form:

$\dot{x}=A x+B v$

The secondary control $v$ can be designed using linear control methods to control the system (4). Figure (1) shows the structure of a control loop in a nonlinear system using linear feedback as a block diagram:

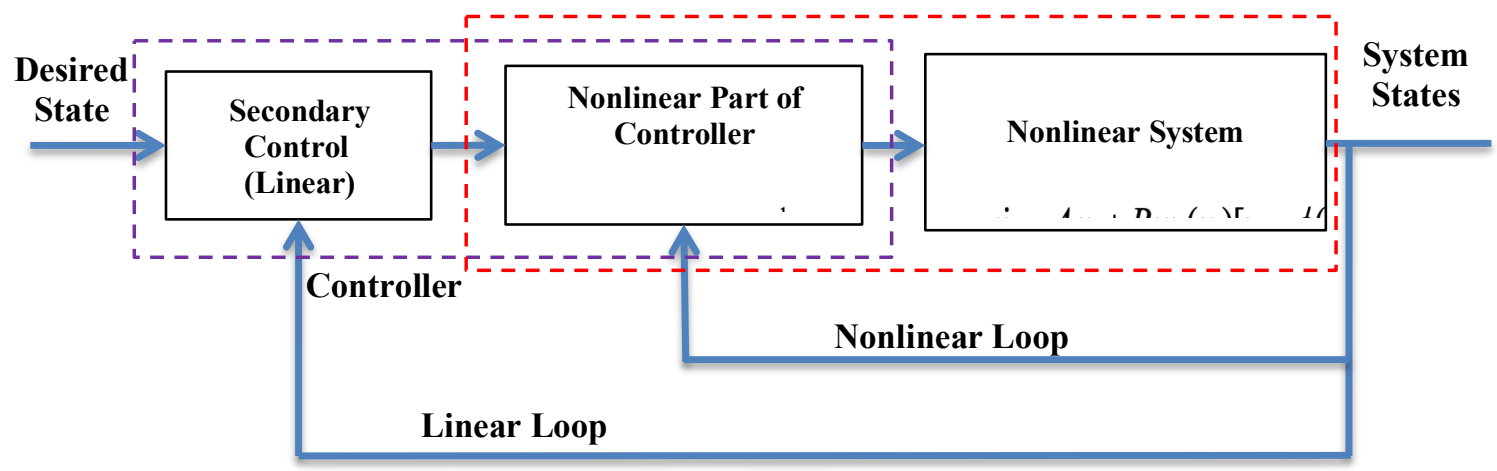

Fig. 1- Nonlinear control loop using linear feedback law

In the linear feedback method, first of all, the nonlinear part of the system is removed by using feedback and the secondary control is designed to control the linearized system. If the system is not in form (2), first, it must find a way to convert the system to form (2) using normalization techniques, and then design the controller. To track the desired output, the input-output feedback linearization theory is used. Consider the following nonlinear system:

$\{\dot{x}=f(x)+g(x) u$

$\{y=h(x)$

$x \in X \subset \mathbb{R}^{n}$ is state vector, $u \in \mathbb{R}$ is control input, $y \in \mathbb{R}$ is system output, $f(x), g(x): D \subset \mathbb{R}^{n} \rightarrow \mathbb{R}^{n}$ and $h(x): D \subset$ $\mathbb{R}^{n} \rightarrow \mathbb{R}$ are nonlinear functions. The issue is to find the control law $b$ that causes the desired output $g$ to be tracked by the system output. For this purpose, first the input-output relevancy will be obtained by deriving from the output of the system. The first output derivative will be as follows:

$\dot{y}=\frac{\partial h}{\partial x} \dot{x}=\frac{\partial h}{\partial x} f(x)+\frac{\partial h}{\partial x} g(x) u$

Definition 3-1 Consider the function $h: D \subset R^{n} \rightarrow R$ and the vector space $f: D \subset R^{n} \rightarrow R^{n} \cdot \frac{\partial h}{\partial x} f(x)$ is a derivative of Lie $h$ relative to $f$ and denoted by $L_{f} h(x)$. Suppose the input appears in the nth derivative of the output as follows:

$y^{(r)}=L_{f}^{r} h(x)+L_{g} L_{f}^{(r-1)} h(x) u$

According to equation (10), the nonlinear functions $f(x)$, $g(x)$ and $h(x)$ must be smooth and their Lie derivatives can be defined. $L_{g} L_{f}{ }^{(r-1)} h(x)$ must be the opposite of zero. Therefore, using the input-output linear feedback theory, the control input is designed as follows:

$u=\frac{1}{L_{g} L_{f}^{(r-1)} h(x)}\left(-L_{f}^{r} h(x)+v\right)$
Using the controller (8) in the system (7), the output differential equation will be converted to a linear form (9).

$y^{(r)}=v$

$v$ can be designed using any linear control method to track the desired output. The stability of internal dynamics after output stabilization should be examined separately from appropriate methods [9]. Suppose that in equation (6) the system multiinput multi-output is nonlinear and is the vector of nonlinear functions of state variables, $g(x)$ is the matrix of nonlinear functions of state variables, $y=h(x)$ is the output vector of system and $\mathrm{u}$ is the input vector. Now, we derive all the outputs for the input to appear.

$\left[\begin{array}{l}y_{1}^{\left(r_{1}\right)} \\ y_{2}^{\left(r_{2}\right)} \\ \vdots \\ y_{m}^{\left(r_{m}\right)}\end{array}\right]=\left[\begin{array}{l}L_{f}^{r_{1}} h_{1}(x) \\ L_{f}^{r_{2}} h_{2}(x) \\ \vdots \\ L_{f}^{r_{m}} h_{m}(x)\end{array}\right]+\left[\begin{array}{l}\sum_{k=1}^{m} L_{g_{k}} L_{f}^{r_{1}-1} h_{1}(x) u_{k} \\ \sum_{k=1}^{m} L_{g_{k}} L_{f}^{r_{2}-1} h_{2}(x) u_{k} \\ \vdots \\ \sum_{k=1}^{m} L_{g_{k}} L_{f}^{r_{m}-1} h_{m}(x) u_{k}\end{array}\right]$
$=L(x)+J(x) u$

The control input vector is designed as follows:

$u=J(x)^{-1}[-L(x)+v]$

The output vector is converted as follows:

$\left[\begin{array}{l}y_{1}^{\left(r_{1}\right)} \\ y_{2}^{\left(r_{2}\right)} \\ \vdots \\ y_{m}^{\left(r_{m}\right)}\end{array}\right]=\left[\begin{array}{l}v_{1} \\ v_{2} \\ \vdots \\ v_{m}\end{array}\right]$

From any linear method, the input vector $\mathrm{v}$ can be determined to stabilize or track the outputs. All v-vector components can be designed separately for output stabilization $[31,32]$. 


\subsection{Extended State Observer}

We use the observer to estimate the unmeasurable information of the system. Now, the controller can be executed by estimated variables and uncertain functions. Using an extended observer, it is possible to estimates of unmeasurable and uncertain parts. In this section, an extended observer that has been presented in the references in recent years is introduced. Consider the nonlinear system in the following form:

$\dot{x}_{1}=x_{2}$

$\dot{x}_{2}=f(x)+g(x) u+d$

$y=x_{1}$

$x$ and $y$ are the state variables, $d(t)$ is the uncertain part and $u$ is the control input. In this system, only the state variable $x$ can be measured. The state observer is defined as follows in reference [37]:

$$
\left\{\begin{array}{l}
\dot{\hat{x}}_{1}=\hat{x}_{2}+\beta_{1} e \\
\dot{\hat{x}}_{2}=f(x)+g(x) u+\hat{d}+\beta_{2} f a l\left(e, \alpha_{1}, \delta\right) \\
\hat{\hat{d}}=\beta_{3} f a l\left(e, \alpha_{2}, \delta\right) \\
\hat{y}=\hat{x}
\end{array}\right.
$$

$e=y-\hat{y}=x_{1}-\hat{x}_{1}$ is estimation error, $\beta_{1}, \beta_{2}, \beta_{3}$ and observer adjustment parameters and $\hat{x}_{1}, \hat{x}_{2}, \hat{d}$ are estimates of variables $x_{1}, x_{2}, d$, respectively. The $\operatorname{fal}(e, \alpha, \delta)$ function is defined as follows:

$f a l(e, \alpha, \delta)=\left\{\begin{array}{l}\frac{e}{\delta^{1-\alpha}},|e| \leq \delta \\ |e|^{\alpha} \operatorname{sign}(e),|e|>\delta\end{array}\right.$

$0<\alpha<1$ and $\delta$ is a small number. Using Equations (15) and (14) in the system (13) the dynamics of the observer error is obtained as follows:

$e_{x_{1}}=\hat{y}-y$

$\dot{e}_{x_{1}}=e_{x_{2}}-\beta_{1} e_{x_{1}}$

$\dot{e}_{x_{2}}=e_{d}-\beta_{2} \operatorname{fal}\left(e_{x_{1}}, \alpha_{1}, \delta\right)$

$\dot{e}_{d}=-\dot{d}(t)-\beta_{3} \operatorname{fal}\left(e_{x_{1}}, \alpha_{2}, \delta\right)$

In reference [37], convergence of error and proof of the stability of this observer has been done.

\subsection{Observer based MIMO feedback linearization}

Here, by combining the contents of the previous sections, the observer based MIMO feedback linearization method for uncertain multi-input multi-output nonlinear systems is described. So consider the following system which is MIMO and nonlinear:

$$
\left\{\begin{array}{l}
\dot{x}=f(x)+g(x) u+w(t) \\
y=h(x)
\end{array}\right.
$$

Where $f(x)$ is a vector of nonlinear functions of state variables, $g(x)$ is a matrix of nonlinear functions of state variables, $y=h(x)$ is a system output vector, $w(t)$ is an uncertain vector, and $u$ is an input vector. Now we derive from each output to display the input:

$$
\begin{aligned}
& {\left[\begin{array}{l}
y_{1}^{\left(r_{1}\right)} \\
y_{2}^{\left(r_{2}\right)} \\
\vdots \\
y_{m}^{\left(r_{m}\right)}
\end{array}\right]=\left[\begin{array}{l}
L_{f}^{r_{1}} h_{1}(x) \\
L_{f}^{r_{2}} h_{2}(x) \\
\vdots \\
L_{f}^{r_{m}} h_{m}(x)
\end{array}\right]+\left[\begin{array}{l}
\sum_{k=1}^{m} L_{g_{k}} L_{f}^{r_{1}-1} h_{1}(x) u_{k} \\
\sum_{k=1}^{m} L_{g_{k}} L_{f}^{r_{2}-1} h_{2}(x) u_{k} \\
\vdots \\
\sum_{k=1}^{m} L_{g_{k}} L_{f}^{r_{m}-1} h_{m}(x) u_{k}
\end{array}\right]+} \\
& {\left[\begin{array}{l}
d_{1}(t) \\
d_{2}(t) \\
\vdots \\
d_{m}(t)
\end{array}\right]} \\
& y^{(r)}=L(x)+J(x) u+D(t)
\end{aligned}
$$

For this nonlinear and uncertain system, the control input vector is designed as follows:

$u=J(x)^{-1}[-L(x)-\widehat{D}(t)+V]$

$V=\left[\begin{array}{l}v_{1} \\ v_{2} \\ \vdots \\ v_{m}\end{array}\right], \quad \widehat{D}(t)=\left[\begin{array}{l}\hat{d}_{1}(t) \\ \hat{d}_{2}(t) \\ \vdots \\ \hat{d}_{m}(t)\end{array}\right]$

Assuming that the uncertain vector is accurately estimated, the output vector is as follows:

$\left[\begin{array}{l}y_{1}^{\left(r_{1}\right)} \\ y_{2}^{\left(r_{2}\right)} \\ \vdots \\ y_{m}^{\left(r_{m}\right)}\end{array}\right]=\left[\begin{array}{l}v_{1} \\ v_{2} \\ \vdots \\ v_{m}\end{array}\right]$

We can determine the input vector $v$ from any linear method to stabilize or track the outputs. The auxiliary input vector Using state feedback technique will be as follows:

$\left[\begin{array}{l}v_{1} \\ v_{2} \\ \vdots \\ v_{m}\end{array}\right]=\left[\begin{array}{l}-k_{11} e_{1}-k_{12} \dot{e}_{1}-\ldots-k_{1 r_{1}} e_{1}{ }^{\left(r_{1}-1\right)}+y_{1_{d}}^{\left(r_{1}\right)} \\ -k_{21} e_{2}-k_{22} \dot{e}_{2}-\ldots-k_{2 r_{2}} e_{2}^{\left(r_{2}-1\right)}+y_{2_{d}}^{\left(r_{2}\right)} \\ \vdots \\ -k_{m 1} e_{m}-k_{m 2} \dot{e}_{m}-\ldots-k_{m r_{m}} e^{\left(r_{m}-1\right)}+y_{m_{d}}^{\left(r_{m}\right)}\end{array}\right]$

According to the uncertain observer design section, each of the uncertainty estimation vector are as follows:

$$
\begin{aligned}
& \left\{\begin{array}{l}
\dot{z}_{i 1}=z_{i 2}+\beta_{i 1} e_{i} \\
\dot{z}_{i 2}=\hat{d}_{i}+L_{f}^{r_{i}} h_{i}(x)+\sum_{k=1}^{m} L_{g_{k}} L_{f}^{r_{1}-1} h_{1}(x) u_{k}+\beta_{i 2} f a l\left(e_{i}, \alpha_{i 1}, \delta_{i}\right) \\
\dot{\hat{d}}_{i}=\beta_{i 3} f a l\left(e_{i}, \alpha_{i 2}, \delta_{i}\right) \\
z_{i 1}=\hat{y}_{i} \\
i=1 \ldots \ldots m
\end{array}\right. \\
& e_{i}=y_{i}-\hat{y}_{i}, \quad \mathrm{i}=1 \ldots \mathrm{m}
\end{aligned}
$$

where $\beta_{i 1}, \beta_{i 2}, \beta_{i 3}$ are observer adjustment parameters and $z_{i 1}, z_{i 2}, \hat{d}_{i}$ are estimates of variables $y_{i}^{\left(r_{i}-1\right)}, y_{i}^{\left(r_{i}\right)}, d_{i}$, respectively. The $\operatorname{fal}(e, \alpha, \delta)$ function is defined as follows: $\operatorname{fal}(e, \alpha, \delta)=\left\{\begin{array}{l}\frac{e}{\delta^{1-\alpha}},|e| \leq \delta \\ |e|^{\alpha} \operatorname{sign}(e),|e|>\delta\end{array}\right.$

$0<\alpha<1$ and $\delta$ is a small number. 


\section{Control system design}

In this section, the observer based MIMO feedback linearization method, is used for the nonlinear two-input twooutput PMSM system. Consider the dynamic equations of the system:

$\left[\begin{array}{c}i_{d} \\ i_{q} \\ \dot{\omega}\end{array}\right]=\left[\begin{array}{c}-\frac{R_{s}}{L} i_{d}+P_{n} \omega i_{q} \\ -P_{n} \omega i_{d}-\frac{R_{s}}{L} i_{q}-\frac{P_{n} \psi_{f}}{L} \omega \\ \frac{P_{n} \psi_{f}}{J} i_{q}-\frac{B}{J} \omega\end{array}\right]+\left[\begin{array}{cc}\frac{1}{L} & 0 \\ 0 & \frac{1}{L} \\ 0 & 0\end{array}\right]\left[\begin{array}{l}u_{d} \\ u_{q}\end{array}\right]+\left[\begin{array}{c}0 \\ 0 \\ -\frac{1}{L}\end{array}\right] T_{L}$

state variables as:

$X=\left[\begin{array}{lll}x_{1} & x_{2} & x_{3}\end{array}\right]=\left[\begin{array}{lll}i_{d} & i_{q} & \omega\end{array}\right]$

Control input vector as:

$U=\left[\begin{array}{ll}u_{1} & u_{2}\end{array}\right]=\left[\begin{array}{ll}u_{d} & u_{q}\end{array}\right]$

Output vector as:

$Y=\left[\begin{array}{ll}y_{1} & y_{2}\end{array}\right]=\left[\begin{array}{ll}i_{d} & \omega\end{array}\right]$

The state space form of this system:

$$
\begin{aligned}
& {\left[\begin{array}{l}
\dot{x}_{1} \\
\dot{x}_{2} \\
\dot{x}_{3}
\end{array}\right]=\left[\begin{array}{c}
-\frac{R_{s}}{L} x_{1}+P_{n} x_{2} x_{3} \\
-P_{n} x_{1} x_{3}-\frac{R_{s}}{L} x_{2}-\frac{P_{n} \psi_{f}}{L} x_{3} \\
\frac{P_{n} \psi_{f}}{J} x_{2}-\frac{B}{J} x_{3}
\end{array}\right]+\left[\begin{array}{ll}
\frac{1}{L} & 0 \\
0 & \frac{1}{L} \\
0 & 0
\end{array}\right]\left[\begin{array}{l}
u_{1} \\
u_{2}
\end{array}\right]+} \\
& {\left[\begin{array}{c}
0 \\
0 \\
-\frac{1}{L}
\end{array}\right] w(t)} \\
& {\left[\begin{array}{l}
y_{1} \\
y_{2}
\end{array}\right]=\left[\begin{array}{l}
x_{1} \\
x_{3}
\end{array}\right]}
\end{aligned}
$$

$w(t)=T_{L}$ is considered as disturbance. In Equation (30) dynamics of a two-input two-output PMSM is nonlinear and uncertain. For this system, we have:

$y^{(r)}=L(x)+J(x) u+D(t)$

$\left[\begin{array}{l}\dot{y}_{1} \\ \ddot{y}_{2}\end{array}\right]=\left[\begin{array}{l}L_{1}(x) \\ L_{2}(x)\end{array}\right]+\left[\begin{array}{ll}J_{11} & J_{12} \\ J_{21} & J_{22}\end{array}\right]\left[\begin{array}{l}u_{1} \\ u_{2}\end{array}\right]+\left[\begin{array}{l}d_{1}(t) \\ d_{2}(t)\end{array}\right]$

$L_{1}(x)=-\frac{R_{s}}{L} x_{1}+P_{n} x_{2} x_{3}$

$L_{2}(x)=\frac{P_{n} \psi_{f}}{J}\left(-P_{n} \omega i_{d}-\frac{R_{s}}{L} i_{q}-\frac{P_{n} \psi_{f}}{L} \omega+\frac{u_{q}}{L}\right)$

$$
-\frac{B}{J}\left(\frac{P_{n} \psi_{f}}{J} i_{q}-\frac{B}{J} \omega\right)
$$

$J_{11}=\frac{1}{L}$

$J_{12}=0$

$J_{21}=0$

$J_{22}=\frac{P_{n} \psi_{f}}{J L}$

$d_{1}(t)=0$

$d_{2}(t)=\frac{B}{J^{2}} T_{L}-\frac{\dot{T}_{L}}{J}$

The control input vector is designed as follows:
$u=J(x)^{-1}[-L(x)-\widehat{D}(t)+V], \quad V=\left[\begin{array}{l}v_{1} \\ v_{2}\end{array}\right], \quad \widehat{D}(t)=$

$\left[\begin{array}{l}0 \\ \hat{d}_{2}(t)\end{array}\right]$

$J(x)^{-1}=\frac{1}{J_{11} J_{22}-J_{12} J_{21}}\left[\begin{array}{cc}J_{22} & -J_{12} \\ -J_{21} & J_{11}\end{array}\right]=\frac{J L^{2}}{P_{n} \psi_{f}}\left[\begin{array}{cc}\frac{P_{n} \psi_{f}}{J L} & 0 \\ 0 & \frac{1}{L}\end{array}\right]$

$L(x)=\left[\begin{array}{l}L_{1}(x) \\ L_{2}(x)\end{array}\right]$

$=\left[\begin{array}{l}-\frac{R_{s}}{L} x_{1}+P_{n} x_{2} x_{3} \\ \frac{P_{n} \psi_{f}}{J}\left(-P_{n} \omega i_{d}-\frac{R_{s}}{L} i_{q}-\frac{P_{n} \psi_{f}}{L} \omega+\frac{u_{q}}{L}\right)-\frac{B}{J}\left(\frac{P_{n} \psi_{f}}{J} i_{q}-\frac{B}{J} \omega\right)\end{array}\right]$

$\widehat{D}(t)=\left[\begin{array}{l}0 \\ \hat{d}_{2}(t)\end{array}\right]=\left[\begin{array}{l}0 \\ \frac{B}{J^{2}} \widehat{T}_{L}-\frac{\dot{\hat{T}}_{L}}{J}\end{array}\right]$

Assuming that the uncertain vector is accurately estimated, the output vector is as follows:

$\left[\begin{array}{l}\dot{y}_{1} \\ \ddot{y}_{2}\end{array}\right]=\left[\begin{array}{l}v_{1} \\ v_{2}\end{array}\right]$

Now the input vector $\mathrm{v}$ can be determined from any linear method to stabilize or track the outputs. For this purpose, the auxiliary input vector using state feedback technique will be as follows:

$\left[\begin{array}{l}v_{1} \\ v_{2}\end{array}\right]=\left[\begin{array}{l}-k_{1} e_{1}+\dot{y}_{1_{d}} \\ -k_{21} e_{2}-k_{22} \dot{e}_{2}+\ddot{y}_{2_{d}}\end{array}\right]$

$e_{1}=y_{1}-y_{1_{d}}$

$y_{1}=i_{d}$

$y_{1_{d}=0}$

$e_{2}=y_{2}-y_{2_{d}}$

$y_{2}=\omega$

$y_{2_{d}=\omega_{\text {ref }}}$

Uncertain variables estimation is obtained as follows:

$$
\begin{aligned}
& \left\{\begin{array}{l}
\dot{z}_{1}=z_{2}+\beta_{1} e \\
\dot{z}_{2}=\hat{d}_{2}+b u_{2}+\beta_{2} \operatorname{fal}\left(e, \alpha_{1}, \delta_{1}\right) \\
\dot{\hat{d}}_{2}=\beta_{3} \operatorname{fal}\left(e, \alpha_{2}, \delta_{2}\right) \\
z_{1}=\hat{y}_{2} \\
b=\left(\frac{P_{n} \psi_{f}}{J}\left(-P_{n} \omega i_{d}-\frac{R_{s}}{L} i_{q}-\frac{P_{n} \psi_{f}}{L} \omega\right)-\frac{B}{J}\left(\frac{P_{n} \psi_{f}}{J} i_{q}-\frac{B}{J} \omega\right)+\frac{P_{n} \psi_{f}}{J L}\right)
\end{array}\right. \\
& \operatorname{fal}(e, \alpha, \delta)=\left\{\begin{array}{l}
\frac{e}{\delta^{1-\alpha}},|e| \leq \delta \\
|e|^{\alpha} \operatorname{sign}(e),|e|>\delta
\end{array}\right. \\
& 0<\alpha<1 \text { and } \delta \text { are small numbers. }
\end{aligned}
$$

\section{Simulation results}

In this section, we simulate the performance of the observerbased MIMO feedback linearization controller in controlling the current and angular speed of a model of a PMSM in the MATLAB. In the next section, the destructive effect of load torque, which is assumed to be uncertain, is first shown. Then, an improvement in the performance of the control system using the disturbance observer is shown. The dynamic model of the PMSM in this section is taken from the reference [3]. The values of the parameters used are listed in Table (1): 
Gh. Omrani, B. Abdi, V. Behnamgol and Gh. Derakhshan./Journal of Engineering Science and Technology Review 14 (3) (2021) 34 - 41

Table 1. Parameter values

\begin{tabular}{c|c|c}
\hline Parameter & Value & unit \\
\hline Rs & 2.875 & Ohm \\
L & 0.085 & H \\
P & 4 & Pair \\
$\psi$ & 0.0175 & Wb \\
B & 1 & Nm \\
J & 0.01 & $\mathrm{Kg}^{*} \mathrm{~m} 2$ \\
\hline
\end{tabular}

Figure (2) shows the load torque.

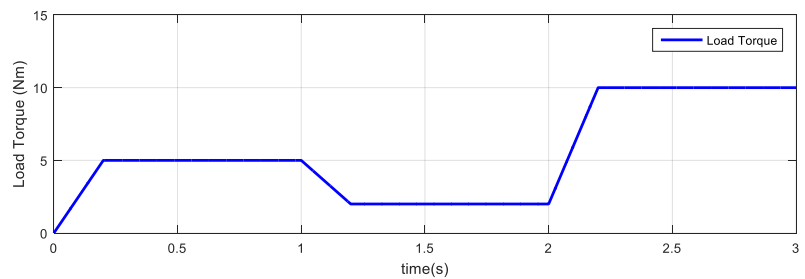

Fig. 2 Load torque
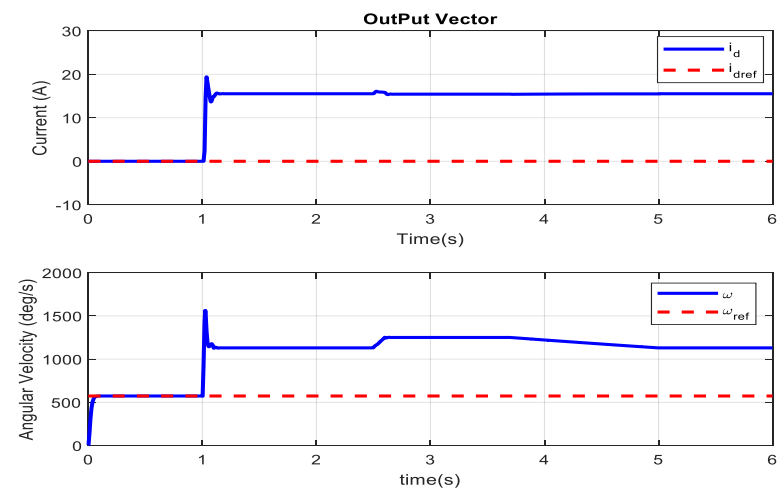

Fig. 3. system outputs by applying the MIMO feedback linearization controller without using load torque estimation

\subsection{Simulation results of control system without using load torque estimation}

In this section, the simulation results of a control system based on MIMO feedback linearization without the use of load torque estimation are shown. In Figure (3), by applying this controller to the motor model, changes in system outputs such as current along the $\mathrm{d}$ axis and angular speed of the shaft are shown. In the initial moments when the load torque value is zero, the system outputs are controlled. But by increasing the amount of load torque and its presence, the outputs have moved away from the desired values. Figure (4) shows the values of the output tracking error. Changes in the control signals issued by the controller in this case are also seen in Figure (5). The control signals are saturated at 250 volts, which is intended to prevent motor damage due to electrical limitations and closer simulations to reality.

\subsection{Simulation results of Load Torque Observer}

In this section, the performance of the observer is investigated. Figure (6) shows the curves of the angular speed of the shaft, the load torque and the uncertain function $\mathrm{d}_{2}$ estimations. It can be seen that these variables are estimated with high accuracy by the observer. Also, the changes in the estimation error of these variables in the initial moments are shown in Figure (7). We see that the estimation has been done in a short time.

\section{3. simulation Results of the control system using load torque estimation}

This section presents the simulation results of the observer based MIMO feedback linearization controller. By applying this controller shown in Figure (8), the outputs such as current along the d-axis and angular speed are displayed. In the initial moments when the load torque value is zero, the outputs are controlled. From the first second, with the presence of load and increasing the amount of load torque, a slight change appears in the outputs but the controller is robust against this disturbance. In Figure (9) the output tracking errors indicate the accuracy and speed of the proposed control system in the presence of disturbance due to load torque. Figure (10) shows the changes in the control signals generated by the controller. The control signals are in the permissible range and the system control is performed without saturating the control inputs.

\section{Conclusion}

In this paper, an observer based multi-input multi-output (MIMO) feedback linearization controller was designed for a PMSM. For this purpose, the load torque is estimated as uncertainty using an extended observer and provided to the controller. The controller is designed as a vector. By simulating the MIMO feedback linearization controller that does not use load torque estimation, it is not able to control the system outputs and the control input signal produced by this control system is saturated. The observer was able to estimate the variables of motor and load torque well and with high accuracy. In the end, it was shown that if the controller uses this estimated information in the feedback, it is able to control the system outputs in the presence of load torque changes.
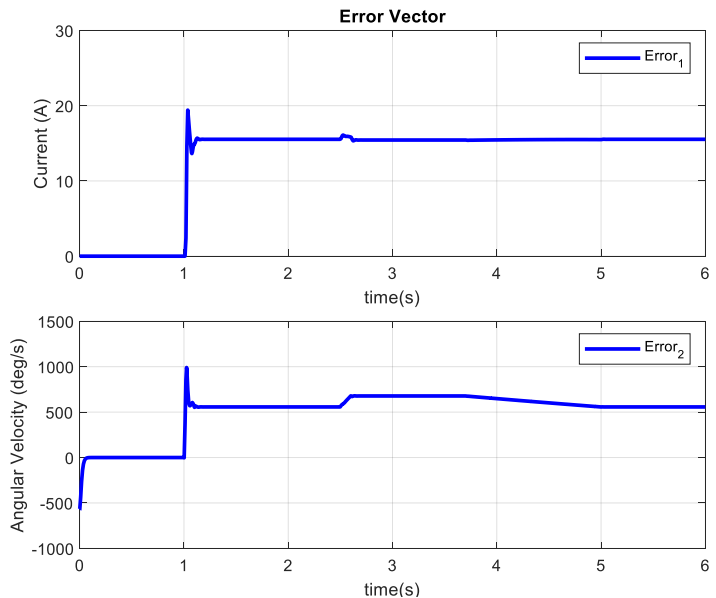

Fig. 4. System outputs tracking error by applying the MIMO feedback linearization controller without using load torque estimation 
Gh. Omrani, B. Abdi, V. Behnamgol and Gh. Derakhshan./Journal of Engineering Science and Technology Review 14 (3) (2021) 34 - 41
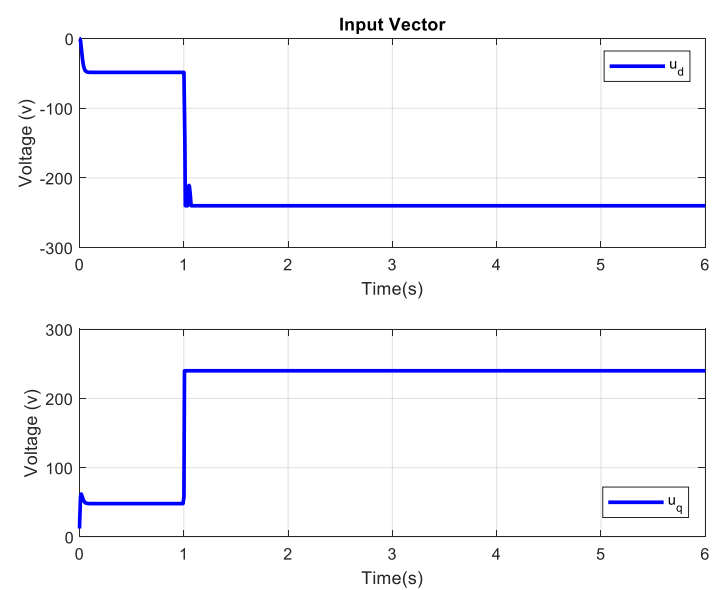

Fig. 5. The input signals by applying the MIMO feedback linearization controller without using load torque estimation
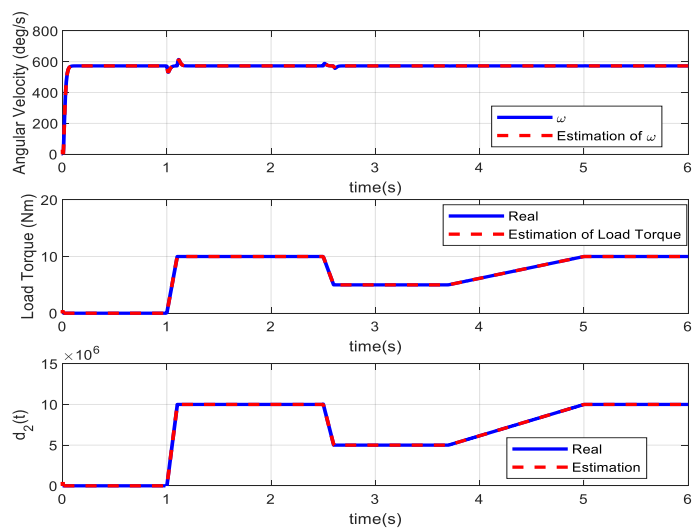

Fig. 6. the angular speed, load torque and uncertain function $\mathrm{d} 2$ and estimation of these variables
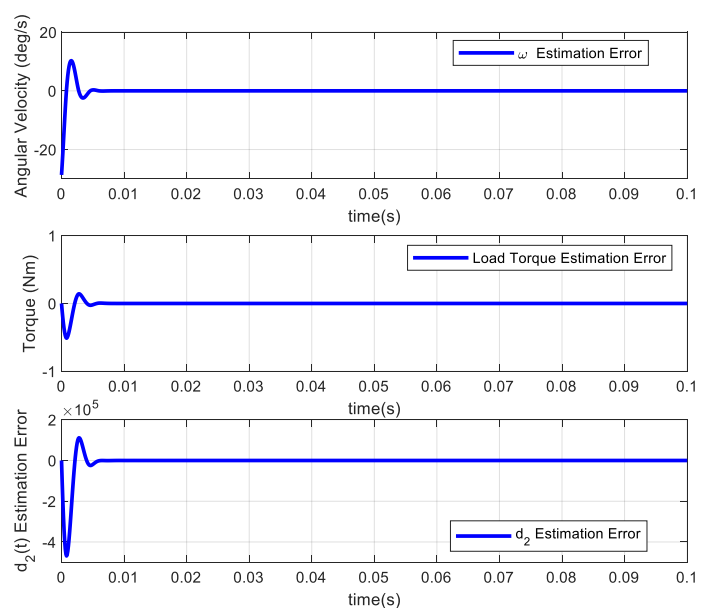

Fig. 7. Estimation error of shaft angular speed, load torque and uncertain function $\mathrm{d} 2$ variables
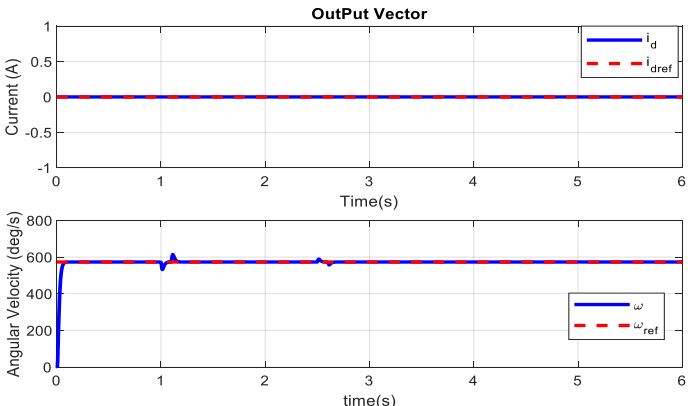

Fig. 8. System output developed by applying the observer based MIMO feedback linearization controller
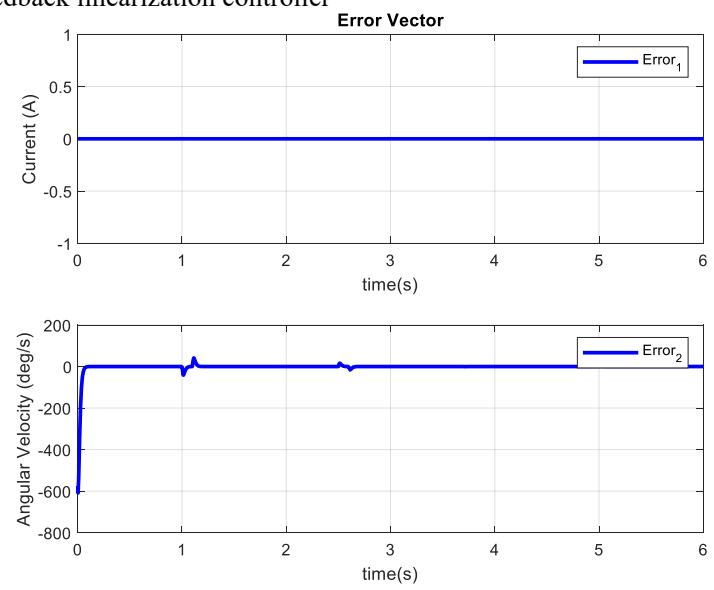

Fig. 9. output tracking error by applying the observer based MIMO feedback linearization controller
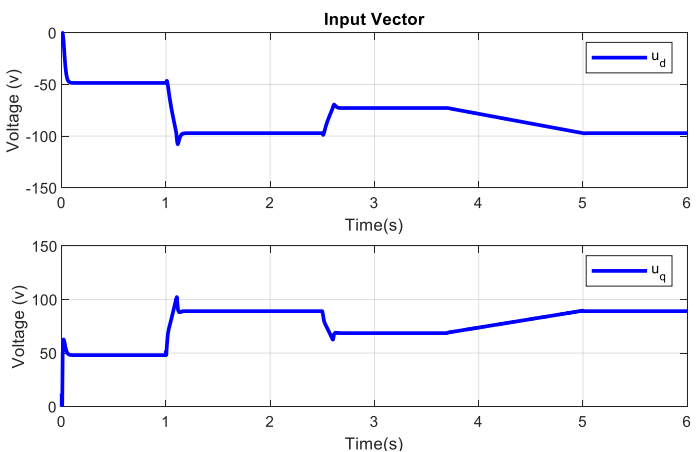

Fig. 10. input signals developed by applying the observer based MIMO feedback linearization controller

This is an Open Access article distributed under the terms of the Creative Commons Attribution License.

\section{(c) (1) (\$)}

\section{References}

1. G. Ram, K. Santha, "Design of an Adaptive Gain variation Sliding Mode Control Algorithm for Extended Non-singular Terminal Sliding Mode Observer based Sensorless PMSM Drive", International Journal of Applied Engineering Research, Vol. 14, No 1, 2019.

2. Kh. Junejo, W. Xu, Ch. Mu, M. M. Ismail, Y. Liu, "Adaptive Speed Control of PMSM Drive System Based A New Sliding-
Mode Reaching Law", IEEE Transactions on Power Electronics, 2020.

3. Parvathy and R. Devanathan, "Linearization of Permanent Magnet Synchronous Motor Using MATLAB and Simulink", INTECH Open Access Publisher, 2011.

4. Bandyopadhyay, F. Deepak, K. S. Kim, "Sliding Mode Control Using Novel Sliding Surfaces", Springer, 2009. 
5. Jong-Woo and L. Sang-Cheol, "Antiwindup Strategy for PI-Type Speed Controller", IEEE Transactions on Industrial Electronics, Vol. 56, 2009.

6. Ch. P. Tana, X. Yub, Zh. Man, "Terminal sliding mode observers for a class of nonlinear systems", Automatica, Vol. 46, 2010.

7. $\mathrm{Xu}$, et al. "Very-low speed control of PMSM based on EKF estimation with closed loop optimized parameters", ISA Transactions, Vol. 52, 2013.

8. F. M. El-Sousy, "High-performance neural-network modelfollowing speed controller for vector-controlled PMSM drive system", IEEE International Conference on Industrial Technology, Vol. 1, 2004.

9. H. J. Marquez, "Nonlinear Control Systems", John Wiley \& Sons Inc, 2003.

10. H. Le-Huy, "Comparison of field-oriented control and direct torque control for induction motor drives", Thirty-Fourth IAS Annual Meeting in Industry, 1999.

11. Boiko and L. Fridman, "Frequency Domain Input-Output Analysis of Sliding-Mode Observers", IEEE Transactions on Automatic Control, Vol. 51, 2006.

12. Omrane, et al. "Modeling and simulation of soft sensor design for real-time speed and position estimation of PMSM", ISA Transactions, Vol. 57, 2015.

13. J. DENG, et al. "Speed Control of Switched Reluctance Motor using Sliding Mode Variable Structure Control", Micromotors Servo Technique, Vol. 7, 2006.

14. J. Liu, H. Li, and Y. Deng, "Torque Ripple Minimization of PMSM based on Robust ILC via Adaptive Sliding Mode Control", IEEE Transactions on Power Electronics, 2017.

15. J. Liu, X. Wang, "Advanced Sliding Mode Control for Mechanical Systems", Springer, 2012.

16. K. Chikh, et al. "Improved DTC Algorithms for Reducing Torque and Flux Ripples of PMSM Based on Fuzzy Logic and PWM Techniques", INTECH Open Access Publisher, 2012.

17. K. Kalsi, J. Lian, S. Huib, S. H. Zak, "Sliding-mode observers for systems with unknown inputs: A high-gain approach", Automatica, Vol. 46, 2010.

18. K. N. Achari, et al. "A Novel MATLAB/Simulink Model of PMSM Drive using Direct Torque Control with SVM", IEEE Multimedia Computing and Systems, 2012.

19. L. Fridman, J. Moreno and R. Iriarte, "Sliding Modes after the First Decade of the 21st Century", Springer, 2011.

20. L. Qi and H. Shi, "Adaptive position tracking control of permanent magnet synchronous motor based on RBF fast terminal sliding mode control", Neurocomputing, Vol. 115, 2013.

21. M. Hajatipour, M. Farrokhi, "Chattering free with noise reduction in sliding-mode observers using frequency domain analysis", Journal of Process Control, 2010.

22. P. Mani, R. Rajan, L. Shanmugam, Y. H. Joo, "Adaptive fractional fuzzy integral sliding mode control for PMSM model", IEEE Transactions on Fuzzy Systems, 2018.
23. P. Vaclavek and P. Blaha, "Synchronous machine drive observability analysis and sensorless control design", IEEE 2nd International in Power and Energy Conference, 2008.

24. S. Coman, et al. "Robust control of a permanent magnet synchronous machine", International Conference on Optimization of Electrical and Electronic Equipment, 2012.

25. S. J. Underwood and I. Husain, "Online Parameter Estimation and Adaptive Control of Permanent-Magnet Synchronous Machines", IEEE Transactions on Industrial Electronics, Vol. 57, 2010.

26. W. $\mathrm{Xu}, \mathrm{Y}$. Jiang, $\mathrm{Ch}$. Mu, "Novel Composite Sliding Mode Control for PMSM Drive System Based on Disturbance Observer", IEEE Transactions on Applied Superconductivity, 2016.

27. X. Liu, H. Yu, J. Yu, L. Zhao, "Combined Speed and Current Terminal Sliding Mode Control with Nonlinear Disturbance Observer for PMSM Drive", IEEE Access, Vol. 4, 2016.

28. X. Xiao, et al. "Performance control of PMSM drives using a selftuning PID", IEEE International Conference on in Electric Machines and Drives, 2005.

29. Y. Jiang, W. Xu, Ch. Mu, Yi. Liu, "Improved Deadbeat Predictive Current Control Combined Sliding Mode Strategy for PMSM Drive System", IEEE Transactions on Vehicular Technology, 2017.

30. Zh. Yin, L. Gong, Ch. Du, J. Liu, and Y. Zhong, "Integrated Position and Speed Loops under Sliding Mode Control Optimized by Differential Evolution Algorithm for PMSM Drives", IEEE Transactions on Power Electronics, 2018.

31. Ch. S. Chiu, "Derivative and integral terminal sliding mode control for a class of MIMO Nonlinear systems", Elsevier, Automatica, Vol. 48, 2012.

32. S. Laghrouche, F. Plestan, A. Glumineau, "Multivariable practical higher order sliding mode control", 44th IEEE Conference on Decision and Control, and the European Control Conference, Seville, Spain, 2005.

33. R. C. Garcia, J. O. P. Pinto, E. A. Batista, L. Galotto, "Design of a MIMO IMC-TS fuzzy speed controller for PMSM", 2017 Brazilian Power Electronics Conference, 2017.

34. Ch. J. Meirinho, A. Bartsch, J. de Oliveira, M. S. M. Cavalca, "An optimal MIMO control approach for PMSM drives", 2017 Brazilian Power Electronics Conference, 2017.

35. N. Maged, "High dynamic performance of PMSM drive using MIMO fuzzy controller", Journal of Electrical Engineering, Vol. $13,2013$.

36. M. Graf, L. Otava, L. Buchta, "Simple Linearization Approach for MPC Design for Small PMSM with Field Weakening Performance", Elsevier IFAC-PapersOnLine, Vol. 48, 2015.

37. H. Yang, Y. Yu, Y. Yuan, X. Fan, "Back-stepping control of twolink flexible manipulator based on an extended state observer", Advances in Space Research, Vol. 56, No. 10, 2015 